\title{
Highly sensitive benzene detection with MOS gas sensors
}

\author{
Martin Leidinger ${ }^{1}$, Tobias Baur ${ }^{1}$, Tilman Sauerwald ${ }^{1}$, Wolfhard Reimringer ${ }^{2}$, Laurent Spinelle ${ }^{3}$, Michel \\ Gerboles $^{3}$, Andreas Schütze ${ }^{1}$ \\ ${ }^{1}$ Saarland University, Campus A5 1, 66123 Saarbrücken, Germany \\ ${ }^{2}$ 3S GmbH, Mainzer Str. 148, 66121 Saarbrücken, Germany \\ ${ }^{3}$ European Commission, Joint Research Centre (JRC), Directorate for Energy, Transport and Climate, \\ Air and Climate Unit, Via Enrico Fermi, 2749, 21027 Ispra (VA), Italy \\ m.leidinger@Imt.uni-saarland.de
}

\begin{abstract}
For detection of benzene, a gas sensor system using metal oxide semiconductor (MOS) gas sensors is presented and measurement results for a concentration range up to $10 \mathrm{ppb}$ are presented. The system is equipped with three gas sensors and advanced temperature control and read-out electronics for using the sensors in temperature cycled operation (TCO). The performance of the system regarding benzene quantification has been tested in two different gas test setups at two different labs. Using features extracted from the TCO signals, regression models for the quantification of benzene have been calculated for both test runs. In the tests with the first setup, benzene was applied in defined gas profiles in a continuous gas flow with variation of humidity and various interferents. In this setup, a quantification accuracy of less than $\pm 0.5 \mathrm{ppb}$ has been achieved at nearly constant background incl. ubiquitous interferent gases; with more complex background variations the quantification error increased to approx. $\pm 2 \mathrm{ppb}$. The second gas mixing system is based on recirculation of the carrier gas stream and closed loop control for the benzene concentration based on continuously available reference data for the benzene concentration. In this system, a similar accuracy was again achieved for low background contaminations and constant humidity; the benzene level could be quantified with an error of less than $0.5 \mathrm{ppb}$.
\end{abstract}

Key words: Indoor air quality, volatile organic compounds, gas sensing, temperature cycled operation, trace gas detection.

\section{Introduction}

Air quality has become an important issue in recent years, especially indoor air quality, as people spend more and more time indoors [1]. Many gaseous substances contribute to decreasing quality of the air we breathe; the most significant are carbon dioxide $\left(\mathrm{CO}_{2}\right)$, carbon monoxide $(\mathrm{CO})$, nitrogen dioxide $\left(\mathrm{NO}_{2}\right)$ and a wide variety of organic substances, summarized as volatile organic compounds (VOCs). In indoor as well as outdoor air, one of the most relevant pollutants is benzene [2]. Due to its toxicity and its carcinogenicity, very low concentrations of benzene should be detected and monitored; threshold limits are in the ppb range [2], e.g. there is a directive by the European parliament suggesting a threshold of $5 \mu \mathrm{g} / \mathrm{m}^{3}$ or $1.6 \mathrm{ppb}$ as long term limit for outdoors [3]. Thus, there is a need for low cost measurement devices able to detect VOCs and especially benzene at these concentrations. We present a gas measurement system designed for this task, based on metal organic semiconductor (MOS) gas sensors and temperature cycled operation (TCO) [4][5].

The system has been tested in two different test facilities using very different methods for generating test gases. In both lab test runs, the goal was quantification of up to $10 \mathrm{ppb}$ of benzene. In the first setup, interferent conditions like changing gas humidity or additional background gases have been added to the test gas.

The presented measurement in the second test setup does not contain gas humidity changes or additional background gases added to the benzene.

\section{Sensor system}

The sensor system is equipped with three different commercial MEMS gas sensors, which are operated in dynamic temperature variation. This approach has already shown promising results for selective detection of $\mathrm{ppb}$ levels of VOCs, even in high interferent gas backgrounds [5]. All sensors are operated and read out independently; rapid temperature 
changes from a high temperature $\left(450{ }^{\circ} \mathrm{C}\right.$ in the first test run and $350{ }^{\circ} \mathrm{C}$ in the second test run) to lower temperatures $\left(300 / 250 / 200{ }^{\circ} \mathrm{C}\right)$ have been performed. The sensor signals, i.e. the logarithm of the conductance of the gas sensing layers, are measured using logarithmic amplifiers, which allow for covering a large signal range, as MOS gas sensor resistances can vary within several orders of magnitude at rapid temperature changes [6][7].

For signal processing, sets of features which describe the shape of the signal (mean value and slope) were extracted from the signals. These features were calculated from several segments of the temperature cycle sensor signal, covering all set temperatures. Using this feature set of each sensor temperature cycle and the known benzene concentration, a PLSR model (partial least squares regression, [8]) is calculated, which generates a linear combination for the features to allow an estimation of the benzene concentration.

\section{Lab 1 measurements and results}

In the first lab characterization of the system, the gas mixing system described in [9] has been used to generate a number of different conditions for the benzene measurement. The system has been specifically designed for trace gas generation. The sensors were placed in a low volume gas stream generated by mass flow controllers, consisting of the carrier air and the added test gases. The benzene concentration has been varied in six steps from 0.5 to $10 \mathrm{ppb}$ for several background and humidity conditions. Starting with only a clean zero air background, two interferent gases ( $\mathrm{CO}$ and toluene) and an additional permanent gas background $\left(\mathrm{CH}_{4}, \mathrm{H}_{2}\right.$, $\mathrm{CO})$ have been added. All variations are listed in Table 1. In each background condition all benzene concentrations were tested.

Table 1: Tested background variations for the benzene measurements in the first set-up

\begin{tabular}{|l|l|l|}
\hline Background & Humidity & Interferents \\
\hline None (zero air) & $\bullet 25 \% \mathrm{RH}$ & $\begin{array}{l}\text { Toluene } \\
\text { ・ } 2 \mathrm{ppb}\end{array}$ \\
\hline $\mathrm{CH}_{4} 1840 \mathrm{ppb} /$ & $\bullet 10 \% \mathrm{RH}$ & Toluene \\
$\begin{array}{l}\mathrm{H}_{2} 500 \mathrm{ppb} / \\
\mathrm{CO} 150 \mathrm{ppb} \\
\text { "ubiquitous } \\
\text { background" }\end{array}$ & $\bullet 25 \% \mathrm{RH}$ & $\bullet 2 \mathrm{ppb}$ \\
{$[10]$}
\end{tabular}

The PLSR results for four selected gas backgrounds are shown in Fig. 1.

In the first case ("A"), two sweeps of the benzene concentration are included, one in pure zero air without interferents and one with a $2 \mathrm{ppb}$ toluene background, at a constant gas humidity of $25 \% \mathrm{RH}$. The benzene concentrations predicted by the PLSR model deviate only slightly from the concentration setpoint. Deviations from the optimal line are lower than $0.5 \mathrm{ppb}$ for all set concentrations, which means sub-ppb accuracy for the system in these nearly ideal conditions.

If more interferent gases are added to the gas mixture and if the gas humidity is varied, the accuracy of the benzene quantification decreases significantly. In cases " $B$ " and " $C$ ", the gas humidity is varied in three steps (see ), and in addition to the measurements in zero air with and without $2 \mathrm{ppb}$ of toluene (scenario " $A$ "), measurements with $\mathrm{CO}$ (350 ppb, "B") and toluene (2 ppb, " $\mathrm{C}$ ") in the permanent gas background mixture were evaluated. In both cases, the accuracy of the predicted concentration is within approx. $\pm 2 \mathrm{ppb}$ of the actual value.

The last presented result ("D") contains only two gas humidities, the signals recorded at the lowest value are not taken into account. However, in addition to the zero air background benzene concentration sweeps, the full set of interferent gases (CO and toluene, all concentrations, with added permanent gas background) at the two remaining humidities was included into the PLSR evaluation. The quality of quantification of benzene is significantly improved over scenarios " $B$ " and " $C$ ", the groups are more compact and deviation from the optimal line is below $1.8 \mathrm{ppb}$ for all concentrations. 

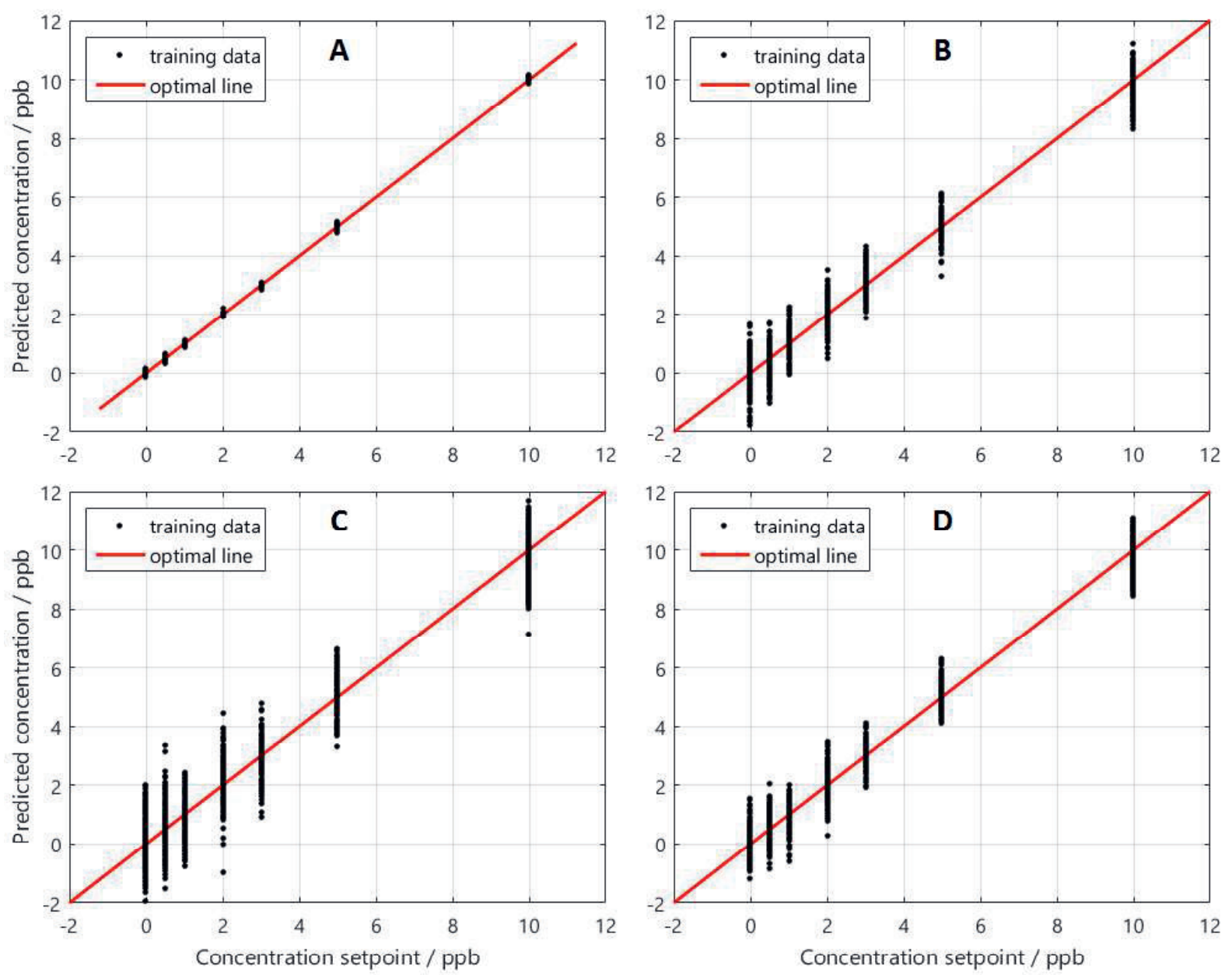

Fig. 1: PLSR benzene quantification results for four different background and interferent configurations:

$A$ : Benzene in pure zero air and with 2 ppb of toluene added, at $25 \% R H$

$B$ : Benzene in pure zero air and ubiquitous background and variation of $\mathrm{CO}$, all humidities

C: Benzene in pure zero air and ubiquitous background and variation of toluene, all humidities

$D$ : Benzene in pure zero air and ubiquitous background and variation of toluene and CO, without lowest humidity

\section{Lab 2 measurements and results}

The second lab characterization has been carried out in the JRC facilities. The exposure chamber described in [11] allowed the generation of multi gas mixtures (with up to 10 compounds) and simultaneously controlling ambient temperature, relative humidity and wind velocity as well as monitoring pressure changes. All parameters are automatically and independently set, measured and controlled. The control is assured by several proportionalintegrative-derivative feed-back loops (PID loops) that requires fast measurements (about $10 \mathrm{~s}$ ) to ensure the efficiency of retroaction on the gas generation system.

It can accommodate several sensors for simultaneous testing with an internal volume of about $120 \mathrm{l}$. Contrary to other exposure chambers, the reference values of all compounds are measured allowing the full traceability to national/international units when evaluating sensors.

Two devices were used to assure the control of the benzene concentration in the exposure chamber. A GC-PID analyzer Syntech Spectras GC955 provided measurements of benzene, toluene, ethylbenzene and xylene every $15 \mathrm{~min}$. throughout the experiment. This GC-PID was calibrated using primary standards gaseous mixture from VSL (The Netherlands). These primary reference gas mixtures were prepared using the gravimetric method in accordance with the ISO 6142:2001 and were contained in $5 \mathrm{~L}$ passivated aluminum gas cylinders. The time resolution requirements for the closed loop control were ensured by Proton Transfer Reaction Mass Spectrometry (PTR-MS). It was set to monitor successively the three BTX 
compounds and 11 variables used to check its suitable working conditions.

In this evaluation, only one of the gas sensors of the systems is considered (AS-MLV, ams), due to technical difficulties with the remaining sensors during the measurement. The presented results show benzene variations between 0 and nearly $8 \mathrm{ppb}$ in a constant background at a humidity of approx. $60 \% \mathrm{RH}$. The signals of the gas sensor at different temperatures and the signals of the two benzene reference measurements (PTR-MS and GC-PID) are shown in Fig. 2.
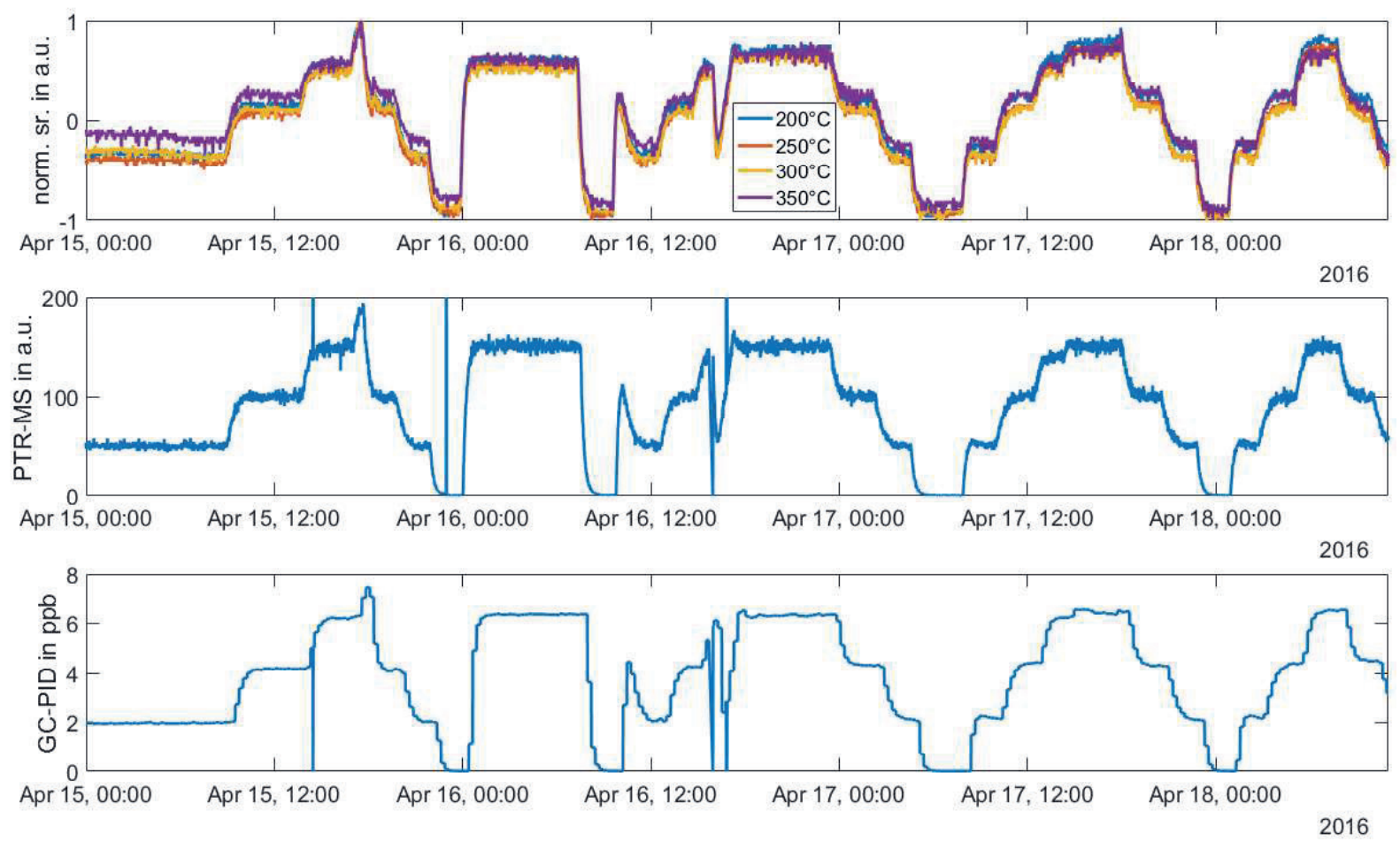

Fig. 2: Sensor signals for the benzene measurement at the second test setup; upper plot: quasi-static sensor signals of the MOS gas sensor for different temperatures of the TCO; middle and lower plots: benzene measurements with PTR-MS (for closed loop control) and GC-PID (traceable reference)

In the upper graph, four quasi-static gas sensor signals are plotted. They represent the four temperatures set points within each temperature cycle. The signals are generated by selecting four points within a cycle (one at each sensor temperature) and plotting the values at these four points for each cycle over the duration of the measurement. Additionally, these sensor signals are mapped to the interval $\{-1,1\}$ for better comparison. The other plots show the benzene control and reference measurements.

For all sensor temperatures the correlation between the gas sensor signal and the reference measurements is very good. The shape of the gas sensor signals and the measured benzene values agree closely. To generate a benzene quantification with the gas sensor, PLSR is also used for this measurement. For this, the recorded data set was divided into three segments, see Fig. 3. The sensor signals from the middle segment (orange line) were used to compute the PLSR model, using the benzene concentration measured by the GC-PID system as a reference for the output values. The data from the other two segments were then evaluated with this model. The calculated benzene concentrations for the gas sensor signals (blue line) are shown as validation in Fig. 3, together with the GC-PID reference data. 


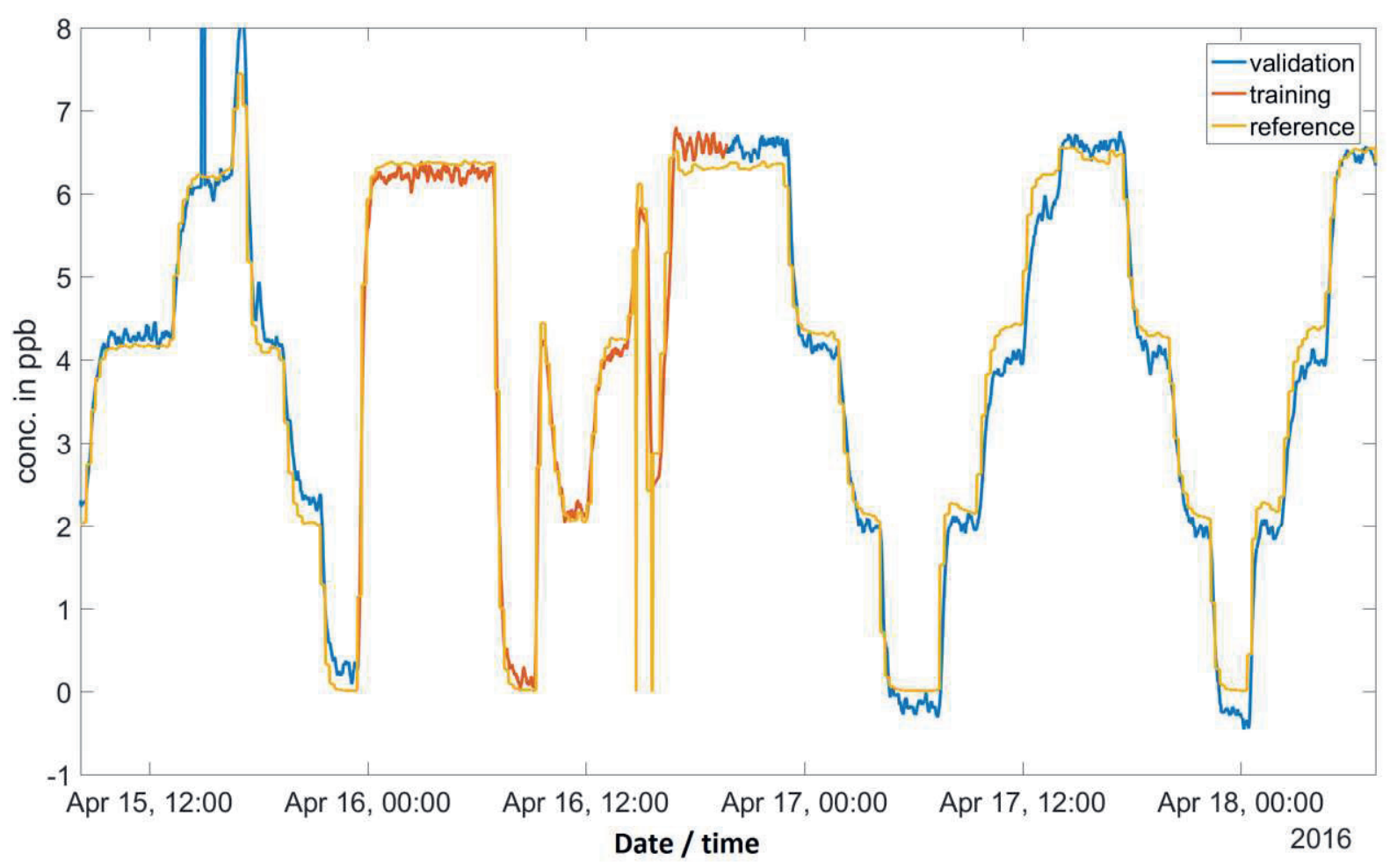

Fig. 3: PLSR evaluation of the benzene measurement at the second test setup. The yellow line shows the benzene concentration as measured by the GC-PID reference. The sensor signals in the middle (orange) segment were used for calculation of the PLSR model, then the full data set was evaluated with this model (orange and blue lines).

For all segments, the benzene concentration calculated from the PLSR model shows very high consistency with the GC-PID results. Except for a few sporadic signal spikes of the MOS sensor signals or the reference measurement signals, the difference of the calculated benzene concentration and the measured concentration is lower than $0.5 \mathrm{ppb}$ throughout all benzene variations. Note that the seemingly noisier sensor signal is similar to the variation of the PTR-MS signal, i.e. reflects the short-time variation of the benzene concentration.

\section{Conclusion}

The presented MOS gas sensor system showed very good performance for benzene quantification, especially at constant gas background conditions and low interferent levels. In two measurements performed at two labs and two very different methods for test gas generation, benzene could be detected in a low concentration range up to $10 \mathrm{ppb}$ with an accuracy of $\pm 0.5 \mathrm{ppb}$ in optimal conditions, i.e. constant humidity and low additional gas background.

Changing the relative humidity and adding a ubiquitous permanent gas background $\left(\mathrm{H}_{2}, \mathrm{CO}\right.$,
$\mathrm{CH}_{4}$ ) plus toluene and varying $\mathrm{CO}$ are added as additional interferent gases decreases the accuracy. However, even in these conditions an accuracy better than $\pm 2 \mathrm{ppb}$ is achieved.

\section{Acknowledgements}

The development of the sensor system and the first sensor tests have received funding from the European Union's Seventh Framework Programme for research, technological development and demonstration under grant agreement No 604311, Project SENSIndoor.

The gas sensor characterization was funded by the European Metrology Research Programme (EMRP), which is jointly funded by the EMRP participating countries within EURAMET and the European Union under the project KEYVOCs.

\section{References}

[1] Klepeis, N. E., Nelson, W. C., Ott, W. R., Robinson, J., Tsang, A. M., Switzer, P., Behar, J. V., Hern, S. and Engelmann, W.: The National Human Activity Pattern Survey (NHAPS): A Resource for Assessing Exposure to Environmental Pollutants, J. Expos. Analysis and Environ. Epidem., 11(3): 231-252, doi:10.1038/sj.jea.7500165, 2001. 
[2] World Health Organization: WHO Guidelines for Indoor Air Quality: Selected Pollutants, Geneva, 2010.

[3] European Parliament, Council of the European Union: Directive 2008/50/EC of the European Parliament and of the Council of 21 May 2008 on ambient airquality and cleaner air for Europe, Off. J. Eur. Union 51, 2008.

[4] Lee, A. P. and Reedy, B. J.: Temperature modulation in semiconductor gas sensing, Sensor. Actuat. B-Chem., 60, 35-42, 1999.

[5] Leidinger, M., Sauerwald, T., Reimringer, W., Ventura, G., and Schütze, A.: Selective detection of hazardous VOCs for indoor air quality applications using a virtual gas sensor array, J. Sens. Sens. Syst., 3, pp. 253-263, doi:10.5194/jsss-3-253-2014, 2014.

[6] Baur. T., Schütze, A. and Sauerwald, T: Optimierung des temperaturzyklischen Betriebs von Halbleitergassensoren, tm - Technisches Messen, 82 (4), 187-195, doi:10.1515/teme2014-0007, 2015.

[7] C. Schultealbert, T. Baur, A. Schütze, S. Böttcher, T. Sauerwald: A novel approach towards calibrated measurement of trace gases using metal oxide semiconductor sensors, Sens. Act. B: Chemical, 239, pp 390-396, doi:10.1016/j.snb.2016.08.002, 2017

[8] Wold, S., Sjöström, M. and Eriksson, L.: PLSregression: a basic tool of chemometrics, Chemometrics and Intelligent Laboratory Systems, 58, 2, pp. 109-130, doi:10.1016/S01697439(01)00155-1, 2001.

[9] Helwig, N., Schüler, M., Bur, C., Schütze, A. and Sauerwald, T.: Gas mixing apparatus for automated gas sensor characterization, Meas. Sci. Technol. 25, 055903, doi:10.1088/09570233/25/5/055903, 2014.

[10] Schütze, A.; Baur, T.; Leidinger, M.; Reimringer, W.; Jung, R.; Conrad T.; Sauerwald, T.: Highly Sensitive and Selective VOC Sensor Systems Based on Semiconductor Gas Sensors: How to?, Environments 2017, 4, 20; doi:10.3390/environments 4010020

[11] Spinelle L., Aleixandre M., Gerboles M.: Report of laboratory and in-situ validation of micro-sensor for monitoring ambient air - Ozone micro-sensors, aSense, model O3-B4, Reports No. EUR 26681. Publications Office of the European Union. http://publications.jrc.ec.europa.eu/repository/han dle/JRC90463, 2014 